\title{
Grid Economics and Business Models
}

\author{
Daniel J. Veit • Wolfgang Gentzsch
}

In Grid Computing systems of heterogeneous networked resources are utilized. Computational resources, storage, memory and bandwidth are complemented with application software and content-oriented input from databases and web services. Hence such systems may operate in predefined and centrally organized ways, or coordination may be conducted in a self-organized, decentralized manner.

Although there are plenty of idle computing resources available on the Internet, only a small percentage of these are actually utilized. One of the reasons for this could be a lack of reliability, availability, quality of service and security. Or the resources are simply not in reach of the user. On the other hand, also the absence of appropriate economic incentives to share resources is possibly a reason.

\footnotetext{
D. J. Veit $(\varangle)$

Dieter Schwarz Chair of Business Administration and Information Systems, Business School,

University of Mannheim, Mannheim, Germany

e-mail: veit@bwl.uni-mannheim.de

\section{W. Gentzsch}

EU Project DEISA, Distributed European Infrastructure for Supercomputing Applications, and Duke University, North Carolina, USA

e-mail: gentzsch@rzg.mpg.de
}

Substantial work has been done on protocols, middleware, and services for security, discovery, sharing, management etc., of computing, storage, data and other resources within dynamic, distributed computing systems. Little attention has been dedicated to the economically efficient allocation of such resources, the embedding of common pricing and business schemes with content provision, as well as the generation of business models based on Grid utilization.

Lately, several initiatives have started in order to generate substantial approaches in this area. The topic has emerged to become a key research issue in the domain of introducing Grid technology to commercial applications. Using Grids for running service oriented architectures and web services may be one concept. Such approaches are fostering a wider commercial adoption of existing research-grade Grid computing systems in the services domain and enable completely novel business models in the future.

In this special issue, we focus on three topics. In the first section, the notion of Grid Economics is developed and outlined. The second section is dedicated to concepts of business models in Grid environments. Observing the development in this field from a wider angle, computational and content oriented services are converging towards one field in which services are in the cen- 
ter of consideration. This domain is covered in the third section. Here, many interdisciplinary research questions meet which cover aspects from computer science, management, economics, law, marketing and game theory, and thus a new cross-institutional discipline - service science - is evolving.

In his featured article, Ramayya Krishnan focuses on the notion of Grid Economics. He picks up at the technical development of Grids within the past five years and draws a circle towards pricing mechanisms for the Grid as well as resource provisioning research. In two distinct scenarios, he addresses key problems while applying economic mechanisms to Grids. In the first scenario, he emphasizes that one of the key problems is the proper judgment of consumers regarding their Grid resource demand. This shortcoming leads to a significant loss in social welfare. In his second scenario, he studies the design of resource allocation mechanisms that can work with stochastic specifications of resource requirements.

Next, Torsten Eymann and coauthors present a framework for trust and reputation in Grid environments. Here, it is assumed that Grid resources are available in a standardized and stable way. Based on this assumption, economic implications can be observed while allocating Grid resources. In their work, the authors propose a concrete reputation-based conceptual framework for enabling future open Grid markets.

Qin Zheng and others elaborate on dynamic load balancing and pricing in Grids. The objective of their mechanism is the determination of algorithms which allow for a minimum response time or minimum computational costs for any new incoming job in the Grid network.

The second featured article by James Broberg and coauthors addresses market-oriented Grids and utility computing and its future development. Here, traditional resource management techniques are investigated and enhanced by adding market-based concepts. Within these, utilitydriven scheduling, 'Catallaxy'-based market approaches and negotiation methodologies are presented.
Roman Beck and his colleagues argue that Grid technology may be appropriate to address resource allocation in departmentalized enterprises. In particular, they propose a pricing mechanism, which allows for a cost-oriented sharing of resources and a utility-centric distribution of resulting prices in such environments.

Kevin Mills and Christopher Dabrowski raise the question whether economics-based resource allocation schemes may improve effectiveness in markets for computational resources. In their investigations, the authors find that a significant added value is measurable through introducing economics-based strategies with respect to the overall resource allocation and the resulting social welfare.

Jim Spohrer and coauthors address the topic of service science in their featured article. They are identifying the relationship between Grid computing and service science and - by deepening the understanding of the notion of resources, protocols, behavior and application domains - enable a better understanding of what role the Grid may play in the development of service science as a discipline.

Dirk Neumann and colleagues elaborate on a framework for commercial Grids, its economic and technical challenges. They identify a catalog of possible market mechanisms to be applied in resource allocation problems in the Grid. Here, they identify both, technical and economic challenges. Moreover, they present results from a concrete simulation setup in which they compare the performance of the individual market mechanisms.

Finally, Werner Streitberger and his coauthors contribute different coordination approaches in simulations of Grid markets. They begin with the dynamic discovery of services and the resulting selection problem. In the identified $n: m$ allocation problem they outline the alternative of using decentralized mechanisms for the solution. Applying their metrics framework, they compare a centralized and decentralized mechanism. In their interpretation they state that the centralized mechanism does not scale in number of participants. 
To this special issue many people have been contributing. First of all we would like to thank Peter Kacsuk and Ian Foster for enabling us producing this volume. Moreover, we would like to thank our reviewers: Christian Becker, Rajkumar Buyya, Clemens van Dinther, Erik Elmroth,
Torsten Eymann, Felix Freitag, Gianfranco Giulioni, Sven Graupner, Sverker Janson, Liviu Joita, Dennis Kundisch, Kevin Lai, Joerg Mueller, Dirk Neumann, Fethi Rabhi, Erel Rosenberg, Stefan Sackmann, Björn Schnizler, Gabriele von Voigt, Kerstin Voss and Tim Weitzel. 